\title{
Statt einer Laudatio: \\ Zum 65. Geburtstag von Karl-Joseph Paquet
}

Lieber Kajo,

vor 30 Jahren habe ich zum ersten Mal Kontakt mit dir aufgenommen. Ich schickte einen Mitarbeiter als Gastarzt zu dir. Wir wollten die Verödung der Ösophagusvarizen bei uns einführen und er sollte von dir lernen.

Mit Helmut Denck bist du ein Pionier der ersten Stunde. Niemand in Deutschland kann das bestreiten, auch wenn du dich in den ersten Jahren gegen so manchen Widerstand im Kollegenkreis durchsetzen musstest.

Wir entdeckten viele Gemeinsamkeiten. So kamen wir beide aus Kliniken, in denen die portale Hypertension ein Schwerpunkt der Abdominalchirurgie war. Du kamst aus der Schule Gütgemann, ich aus der Göttinger Schule von Koncz und wir hatten als Chirurgen gegenüber unseren internistischen Kollegen den Vorteil, dass wir beides anbieten konnten, die Verödungstherapie und die Shuntchirurgie. Wir tauschten unsere Erfahrungen aus und verfolgten in vielen Bereichen der Chirurgie die gleichen Ziele. Aber das allein war wohl nicht die Ursache, warum Freundschaft entstand. Wir hatten und haben übereinstimmende Vorstellungen darüber, dass zwischen dem technischen Fortschritt und einer humanen Medizin ein Konsens gefunden werden muss. Beide gehören wir der Generation an, in der noch eine umfassende chirurgische Ausbildung in mehreren Bereichen möglich war. Du hast 1964 an der Universität Bonn das Staatsexamen gemacht, promoviert und nach der Habilitation in der Bonner Klinik neben der Allgemeinchirurgie die Anerkennung als Gefäßchirurg und Unfallchirurg erworben.

Die Endoskopie wurde in der gastroenterologischen Chirurgie in vielen diagnostischen und therapeutischen Bereichen unverzichtbar. Zusammen mit Helmuth Deck hast du den Plan einer Zeitschrift Endoskopie in der Chirurgie entwickelt, die in meinem kleinen medizinischen TM-Verlag erscheinen sollte, aber im weiteren Verlauf wurde uns klar, dass dieses

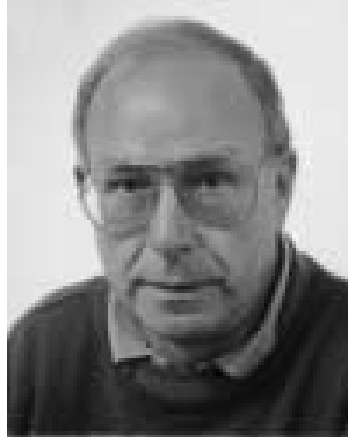

Thema nur im größeren Rahmen behandelt werden konnte. War schon dieser Verlag ein großes Wagnis, so wurde die Einführung einer neuen Zeitschrift vollends zum finanziellen Risiko. Ich wundere mich heute noch über unseren Mut. Wir gründeten die Chirurgische Gastroenterologie mit interdisziplinären Gesprächen. Im April des Jahres 1984 haben wir im Anschluss an den Chirurgenkongress zu viert in einem kleinen Nebenzimmer des Hotels an der Oper in München unsere Zeitschrift aus der Taufe gehoben. Kurt Kanis, mein Kompagnon im TM-Verlag, war mit von der Partie. Bei der nächsten Sitzung kam Aristaks Akovbiantz hinzu, den du als Vertreter der Schweiz zur Mitarbeit gewonnen hattest. Das Konzept war klar umrissen. In jeder Nummer der Zeitschrift sollte ein Gebiet der Abdominalchirurgie unter Mitarbeit von Internisten, Radiologen und Pathologen umfassend behandelt werden. Wären wir nicht immer wieder zu ganz spontanen Entschlüssen gekommen, hätte wir es wohl kaum geschafft. Wir hatten eine Menge guter Einfälle und setzten sie bereits in dieser ersten Sitzung in die Tat um. Helmuth Denck rief kurz entschlossen Ernst Bodner in Innsbruck an und bat ihn, die redaktionelle Leitung der ersten Nummer mit dem Thema «Papilla Vateri» zu übernehmen. Er hat zugesagt, obwohl er für diese erste Nummer nur 8 Monate Zeit hatte. Ich erinnere mich, wie wir während des Telefongesprächs im Gesicht von Helmuth Denck ablesen wollten, ob er erfolgreich wäre. Ernst Bodner sagte spontan zu.

Wir arbeiteten alle außerhalb des Universitätsbereichs und hatten zwei wesentliche Ziele im Auge. Erstens wollten wir den Chirurgen in außeruniversitären Krankenhäusern mehr Gehör verschaffen, denn es gibt keinen Zweifel daran, dass diese in der medizinischen Öffentlichkeit viel zu wenig repräsentiert waren, obwohl ihre Erfolge auch bei der Entwicklung neuer Verfahren sich durchaus mit Universitätskliniken messen konnten. Wir hatten die Erfahrung gemacht, dass viele Bemühungen um Veröffentlichung wesentlicher neuer Ver-

\section{KARGER}

Fax +497614520714

E-mail Information@Karger.de www.karger.com/journals/cga

(C) 2002 S. Karger GmbH, Freiburg 
fahren und Erfahrungen in führenden Zeitschriften und auf Kongressen unterdrückt wurden. Zweitens wollten wir mit dieser Zeitschrift dem Ziel einer interdisziplinären Gastroenterologie näher kommen. Bei der Lösung vieler großer Probleme der Medizin und bei der Bewältigung des Fortschritts spielen die Indikationen und somit die interdisziplinäre Koordination eine entscheidende Rolle. Wir haben beide erfahren, dass diese interdisziplinäre Zusammenarbeit große Anforderungen an Toleranz und auch manchen Verzicht bedeutet, du im Heinz-Kalk-Krankenhaus in Bad Kissingen, ich in einer neu gegründeten interdisziplinären gastroenterologischen $\mathrm{Ab}$ teilung. Das alles ist nun Vergangenheit.
Ich verfolge als Mitbegründer die weitere Entwicklung unserer Zeitschrift mit großem Interesse. Mir erscheint es sinnvoll, der nächsten Generation zu erzählen, was sich in den vergangenen 50 Jahren in der Chirurgie ereignet hat. Vielleicht ist das ein neuer Themenkreis unserer Zeitschrift. In einem solchen Rückblick könnte deutlich gemacht werden, wie sich nicht nur die diagnostischen und operativen Methoden, sondern auch die Wertvorstellungen in unserem ärztlichen Beruf geändert haben.

In alter Verbundenheit dein Erasmus Zöckler 